\title{
Osteopontin Peptide Icosamer Containing RGD and SLAYGLR Motifs Enhances the Motility and Phagocytic Activity of Microglia
}

\author{
Il-Doo Kim ${ }^{1,2}$, Hahnbie Lee ${ }^{1,2}$, Yin-Chuan Jin ${ }^{3}$ and Ja-Kyeong Lee ${ }^{1,2 *}$ \\ ${ }^{1}$ Department of Anatomy, ${ }^{2}$ Medical Research Center, Inha University School of Medicine, Incheon 22212, Korea, \\ ${ }^{3}$ Department of Histology and Embryology, Binzhou Medical University, Yantai 264000, China
}

\begin{abstract}
Osteopontin (OPN) is a secreted glycoprotein that is expressed in various tissues, including brain, and mediates a wide range of cellular activities. In a previous study, the authors observed the robust neuroprotective effects of recombinant OPN and of RGD and SLAYGLR-containing OPN-peptide icosamer (OPNpt20) in an animal model of transient focal ischemia, and demonstrated antiinflammatory and pro-angiogenic effects of OPNpt20 in the postischemic brain. In the present study, we investigated the effects of OPNpt20 on the motility and phagocytic activity of BV2 cells (a microglia cell line). F-actin polymerization and cell motility were significantly enhanced in OPNpt20-treated BV2 cells, and numbers of filopodia-like processes increased and lamellipodia-like structures enlarged and thickened. In addition, treatment of cells with either of three mutant OPN icosamers containing mutation within RGD, SLAY, or RGDSLAY showed that the RGD and SLAY motifs of OPNpt20 play critical roles in the enhancement of cell motility, and the interaction between exogenous OPNpt20 and endogenous av and a4 integrin and the activations of FAK, Erk, and Akt signaling pathways were found to be involved in the OPNpt20-mediated induction of cell motility. Furthermore, phagocytic activity of microglia was also significantly enhanced by OPNpt20 in a RGD and SLAY dependent manner. These results indicate OPNpt20 containing RGD and SLAY motifs triggers microglial motility and phagocytic activity and OPNpt20-integrin mediated signaling plays a critical role in these activities.
\end{abstract}

Key words: osteopontin icosamer, BV2, RGD, SLAYGLR, motility, phagocytosis

\section{INTRODUCTION}

Osteopontin (OPN) is a glycoprotein expressed in macrophages/ microglia and reactive astrocytes in the CNS [1,2], and under pathological conditions, its up-regulation has been reported to

Received July 31,2017, Revised November 16, 2017 ,

Accepted December 1,2017

* To whom correspondence should be addressed.

TEL: 82-32-860-9893, FAX: 82-32-884-2105

e-mail:jklee@inha.ac.kr have protective and anti-inflammatory effects. For example, in the subarachnoid hemorrhagic brain, recombinant OPN prevented cerebral vasospasm by inducing MAPK phosphatase (MKP)-1 (an endogenous MAPK inhibitor) and decreasing the apoptotic response [3]. OPN is also expressed in substantia nigra, and in an animal model of Parkinson's disease and its interactions with $\alpha v, \beta 3$ and $\beta 1$ integrin and CD44 receptors had neuroprotective effects [4]. Protective effects of OPN have also been reported in animal models of stroke, for example, in a photothrombotic stroke model, a transient middle cerebral artery occlusion (MCAO) model, and a neonatal model of hypoxic-ischemic brain injury [5-
Copyright (c) Experimental Neurobiology 2017. www.enjournal.org
This is an Open Access article distributed under the terms of the Creative Commons Attribution Non-Commercial License (http://creativecommons.org/licenses/by-nc/4.0) which permits unrestricted non-commercial use, distribution, and reproduction in any medium, provided the original work is properly cited. 
7]. Although, OPN deficient mice exhibited retrograde degeneration of the ipsilateral thalamus but no increase in infarct volume in a photothrombotic stroke model [5], the administration of exogenous OPN reduced infarct volumes and improved long-term neurological functions in a neonatal model of hypoxic-ischemic brain injury [6]. We have also observed the robust neuroprotective effects of recombinant OPN in a rat MCAO model, and reported its neuroprotective potency was markedly enhanced when recombinant OPN was delivered encapsulated in biodegradable gelatin microspheres [7].

OPN has two specific integrin binding motifs, that is, an arginine-glycine-aspartic acid (RGD) and a serine-leucine-alaninetyrosine-glycine-leucine-arginine (SLAYGLR) motifs. Its RGD motif binds to $\alpha v \beta 3$, $\alpha v \beta 5$, $\alpha v \beta 1$, and $\alpha 8 \beta 1$ [8] and its SLAYGLR motif, which is exposed by thrombin cleavage, binds to $\alpha 9 \beta 1$ and a $4 \beta 1[9,10]$. Doyle [11] found that 20- or 10-amino acid OPNpeptides containing the RGD and SLAYGLR motifs had robust neuroprotective effects in a MCAO animal model, and Iczkiewicz [12] showed that a 15-amino acid RGD-containing peptide had neuroprotective properties in an animal model of Parkinson's disease. In a previous study, we also showed a 20 amino acid OPNpeptide containing the RGD and SLAYGLR motifs (OPNpt20) had robust neuroprotective effects in the ischemic rat brain [13] and that its anti-inflammatory and pro-angiogenic effects might underlie these effects $[13,14]$. In our previous studies, the importance of the RGD and SLAYGLR motifs were confirmed by comparing the protective effects of three mutant peptides, in which RGD, SLAY, or RGDSLAY motifs were replaced by RAA, SLAA, or RAASLAA, respectively $[13,14]$.

Microglia are key mediators of immune surveillance and inflammatory response in the central nervous system (CNS) [15]. Under pathological conditions, microglia secrete anti-inflammatory cytokines and neurotrophic factors, but they also secrete proinflammatory cytokines that cause secondary damages in brain tissue due to neurotoxin accumulation and subsequent neurodegeneration $[15,16]$. Although under normal conditions, microglia are motile and perform immune surveillance role [16], when activated, they are markedly more motile and numbers and lengths of filopodia- and lamellipodia-like structures are much increased [17]. Under pathological conditions, ramified microglia migrate to regions of injury and remove pathogens and dead cell debris by recognizing phosphatidylserine on their membranes [1]. OPNmediated cell migration and phagocytosis have been reported in different cell types, in particular, OPN remarkably enhanced neutrophil and macrophage migration via an integrin/FAK and CD44 signaling pathway $[18,19]$. Schack [20] found OPN-coated synthetic beads were phagocytosed by monocytes via $\alpha x \beta 2$ integrin pathways, and Heilmann [21] showed the impaired phagocytosis of macrophages from OPN-deficient mice was rescued by recombinant OPN treatment.

In the present study, we investigated whether OPNpt20 is also able to induce the motility and phagocytic activity of microglia and examined the molecular mechanism responsible using the above-mentioned three mutant peptides.

\section{MATERIALS AND METHODS}

\section{Cell culture}

BV2 cells, a murine microglia cell line, were grown in Dulbecco's modified Eagle’s medium (DMEM; Gibco, Carlsbad, CA), supplemented with $1 \%$ penicillin and streptomycin (Gibco, Carlsbad, CA) and 5\% fetal bovine serum (FBS) (Gibco, Carlsbad, CA) at $37^{\circ} \mathrm{C}$ in a $95 \%$ air $/ 5 \% \mathrm{CO}_{2}$ humidified atmosphere. Cells were prepared in 24-well tissue culture plates (Corning, Corning, NY) at $2 \times 10^{4}$ cells/well.

\section{Peptide treatment}

BV2 cells were incubated with OPN-peptide icosamer (OPNpt20) (0.5 or $1 \mu \mathrm{g} / \mathrm{ml}$; Peptron, Daejon, South Korea) or three mutant OPNpt20 peptides (OPNpt20-RAA, OPNpt20-SLAA and OPNpt20-Db) (1 $\mu \mathrm{g} / \mathrm{ml}$; Peptron, Daejon, South Korea) for $6 \mathrm{hrs}$ at $37^{\circ} \mathrm{C}$. Fibroblast growth factor (FGF) $(1 \mu \mathrm{g} / \mathrm{ml}$; Sigma Aldrich, St. Louis, MO) and recombinant human OPN (rhOPN) $(1 \mu / \mathrm{ml}$; Atgen, Seongnam, South Korea) were used as positive controls, and cytochalasin B (CytB) $(1 \mu \mathrm{g} / \mathrm{ml}$; Sigma Aldrich, St. Louis, MO) was used as a negative control. For inhibition assays, BV2 cells were incubated with $1 \mu \mathrm{g} / \mathrm{ml}$ of OPNpt20 for 6 hrs in the presence or absence of $0.2 \mu \mathrm{M}$ wortmannin (pharmacological inhibitor of Erk; Calbiochem, San Diego, CA) or 20 M PB98059 (pharmacological inhibitor of Akt; Calbiochem, San Diego, CA). Cell images were examined by live cell imaging system or confocal microscopy at the indicated time points.

\section{Live cell imaging analysis}

To observe cell morphologies and motilities, time-lapse images were recorded using the Juli stage (NanoEnTek Inc., Seoul, South Korea), according to the manufacturer's instructions. Mean migration distances and speeds were obtained using the Image J program.

\section{Phalloidin staining}

After treatment with each peptide for 6 hrs, cells were washed with sterile PBS, fixed using 4\% paraformaldehyde for $10 \mathrm{~min}$, washed twice, and stained by adding FITC-labeled phalloidin 
(Thermo Fisher Scientific, Waltham, MA) in sterile PBS for 40 mins. Cells were then mounted with Vectashield mounting medium containing DAPI (Vector Laboratories, Burlingame, CA) and images were obtained by confocal microscopy (Zeiss LSM 510 Meta, Jena, DE). Fluorescence intensities and cell areas were measured using the Image J program.

\section{Phagocytosis assay}

For phagocytic assays, BV2 cells were stimulated with FITClabeled zymosan particles (Molecular Probes, Eugene, OR) and time-lapse images were recorded by live cell imaging system. Phagocytosis was assessed as percentages of phagocytic cell and of phaogocytosed particles, and mean numbers of particle per cell by counting 10 different fields of photomicrographs at indicated times after peptide treatment.

\section{Immunoblot analysis}

Cells were lysed with RIPA buffer (50 mM Tris-HCl (pH 7.4), 1\% NP40, 0.25\% sodium-deoxycholate, $150 \mathrm{mM} \mathrm{NaCl}$ ) containing complete Mini protease inhibitor cocktail tablet (Roche diagnostics, Basel, Switzerland) and phosphatase inhibitor cocktail tablet (Roche diagnostics, Basel, Switzerland). Lysates were centrifuged at $14,000 \mathrm{~g}$ for $15 \mathrm{mins}$ at $4^{\circ} \mathrm{C}$, and proteins $(20 \mu \mathrm{g})$ were separated in $10 \%$ sodium dodecyl sulfate-polyacrylamide gels. After blocking the membranes so obtained with 5\% non-fat milk for $1 \mathrm{hr}$, they were incubated with primary antibodies diluted 1:1000 for anti-FAK, anti-pFAK (Santa Cruz Biotechnology), anti-Erk, antipErk, and anti-Akt, or 1:2000 for anti-pAkt (Cell Signaling, Danvers, MA) and anti- $\boldsymbol{\alpha}$-tubulin (1:2000, Millipore, Billerica, MA) overnight at $4^{\circ} \mathrm{C}$. Blots were detected using a chemiluminescence kit (Roche, Basel, Switzerland) using anti-rabbit and anti-mouse HRP-conjugated secondary antibodies (1:5000, Santa Cruz Biotechnology).

\section{Pull-down assay}

Biotin pull-down assays were performed using $20 \mu \mathrm{l}$ (50\% slurry) of streptavidin-agarose beads (Pierce, Rockford, IL). Biotinylated OPNpt20 (1 $\mu \mathrm{g} / \mathrm{ml}$; Peptron, Daejon, South Korea) was incubated with BV2 whole cell proteins for $6 \mathrm{hrs}$ at $4^{\circ} \mathrm{C}$ with rotation, and then streptavidin-agarose beads were added and incubated for $1 \mathrm{hr}$. After centrifuging at 6,000 $\mathrm{g}$ for $1 \mathrm{~min}$, washing 3 times, the analysis was performed by immunoblotting with anti-integrin alpha v (1:2000, Abcam, Cambridge, UK), anti-integrin a4 (1:1000, Santa Cruz Biotechnology), and anti- $\alpha$-tubulin (1:5000, Millipore, Billerica, MA) antibodies.

\section{Statistical analysis}

Two-sample comparisons were performed using the Student's t test and multiple comparisons by one-way analysis of variance (ANOVA) followed by Newmane-Keuls post hoc test. Results are presented as means \pm SEMs and statistical difference was accepted at the $5 \%$ level.

\section{RESULTS}

\section{RGD-containing osteopontin-peptide icosamer enhanced microglia motility}

BV2 cells (a microglial cell line) were treated with rhOPN (1 $\mu \mathrm{g} /$ $\mathrm{ml}$ ) and monitored for motility over 6 hrs using a live cell imaging system. Mean total migration distance of BV2 cells treated with rhOPN was found to be significantly greater than that of PBStreated controls, but slightly less than that of cells treated with FGF ( $1 \mu \mathrm{g} / \mathrm{ml}$; a well-known inducer of cell migration) [22] (Fig. 1B D, $J$ and Movie S1). When cells were treated with osteopontin-peptide icosamer harboring RGD and SLAYGLR motifs (OPNpt20, $1 \mu \mathrm{g}$ / $\mathrm{ml}$; Fig. 1A) for $6 \mathrm{hrs}$, mean total migration distance increased to $176.3 \pm 6.1 \%$ of that of PBS-treated controls (Fig. 2E, J and Movie S2). While a moderate motility increase was detected in OPNpt20RAA $(1 \mu \mathrm{g} / \mathrm{ml}$, RGD replaced with RAA) or OPNpt20-SLAA (1 $\mu \mathrm{g} / \mathrm{ml}$, SLAY replaced with SLAA)-treated cells (134.9 \pm 5.4 and $145.4 \pm 7.2 \%$, respectively), no such increase was observed for OPNpt20-Db ( $1 \mu \mathrm{g} / \mathrm{ml}$, RGDSLAY replaced with RAASLAA)treated cells (Fig. 2F H, J). In addition, mean speeds for each hour from 1 to 6 hrs after treating cells with OPNpt20 were significantly greater than those of PBS-treated controls and those of cells treated with the three mutant peptides (Fig. 1K). Induction of cell motility of microglia was further confirmed by primary microglial cultures (Supplement Fig. 1 and Movie S3). However, mean total distances migrated and mean speeds of CytB $(1 \mu \mathrm{g} / \mathrm{ml})$-treated BV2 cells were significantly less than those of PBS-treated controls (Fig.1I K). These results show OPNpt20 induced microglial cell motility and that the RGD and SLAY motifs of OPNpt20 contributed to this process.

\section{OPNpt20 increased F-actin polymerization and distribution}

The observed OPNpt20-mediated induction of BV2 cell motility prompted us to examine the effects of OPNpt20 on F-actin polymerization and morphological changes in BV2 cells. Cells were incubated with OPNpt20 $(0.5$ or $1 \mu \mathrm{g} / \mathrm{ml})$ or OPNpt20-Db $(1 \mu \mathrm{g} / \mathrm{ml})$ for 6 hrs and then stained with FITC-labeled phalloidin, which has been widely used to visualize F-actin. Under normal culture conditions, BV2 cells were round and displayed many filopodia-like processes and long protrusions (Fig. 2A), but after 

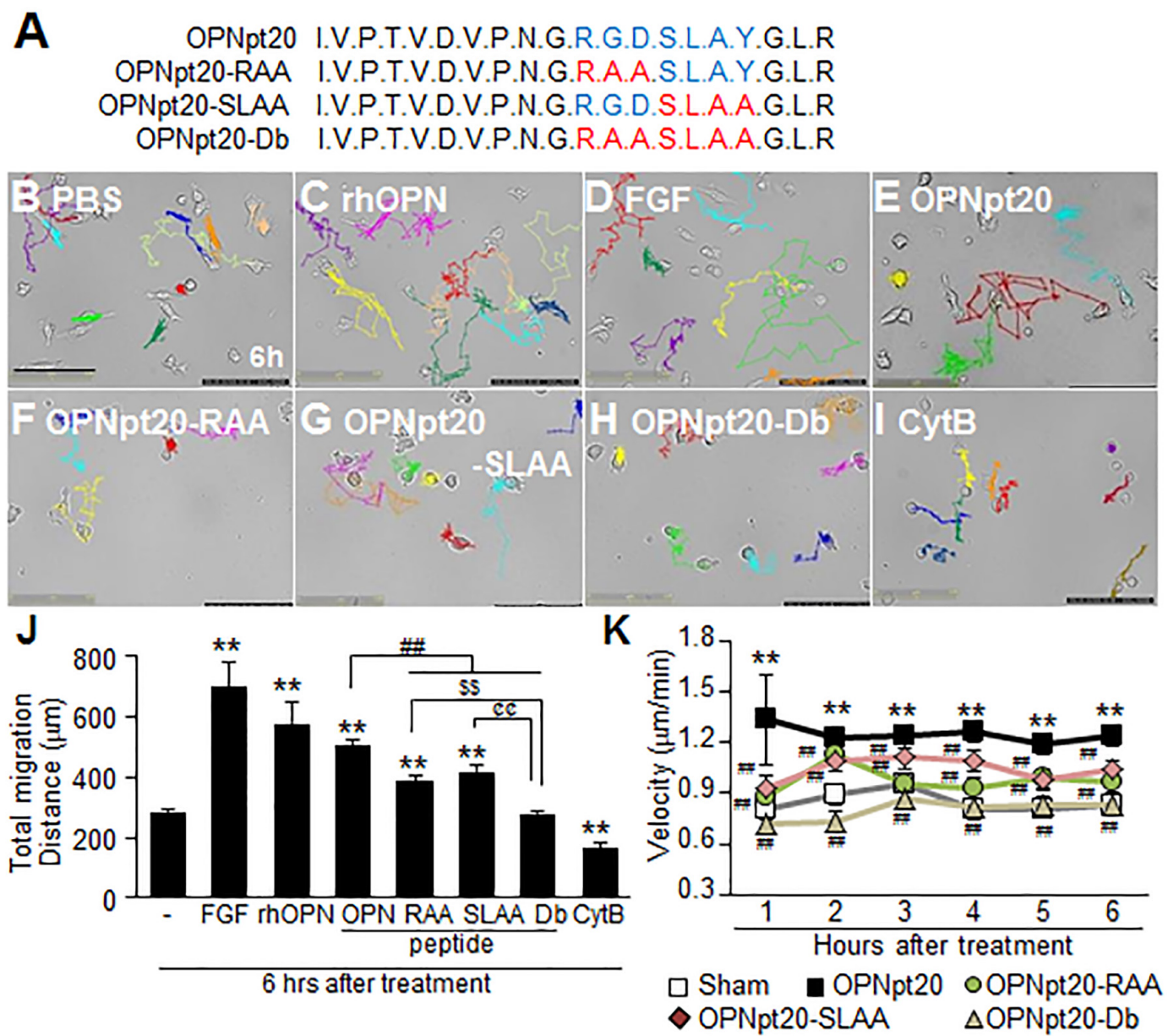

Fig. 1. Induction of microglial cell motility by OPNpt20. (A) Sequences of OPNpt20 and of the three mutant peptides. (B I) BV2 cells were treated with FGF (C, $1 \mu \mathrm{g} / \mathrm{ml})$, rhOPN (D, $1 \mu \mathrm{g} / \mathrm{ml})$, OPNpt20 (E, $1 \mu \mathrm{g} / \mathrm{ml})$, three mutant peptides (F $\mathrm{H}, 1 \mu \mathrm{g} / \mathrm{ml}$ each), or CytB (I, $1 \mu \mathrm{g} / \mathrm{ml})$ and cell motilities were monitored using a live cell imaging analyzer every $10 \mathrm{~min}$ for $6 \mathrm{hrs}$. Motile trajectory of each cell for 6 hrs are presented (B I) and mean total migration distances over 6 hrs $(J)$ and mean hourly migration speeds $(K)$ are presented as means \pm SEMs $(n=20) .{ }^{* *} \mathrm{p}<0.01$ versus PBS-treated controls, ${ }^{\# *} \mathrm{p}<0.01$, ${ }^{\$ s} \mathrm{p}<0.01,{ }^{4 c} \mathrm{p}<0.01$ between indicated groups. Scale bars represent $125 \mu \mathrm{m}$.

rhOPN treatment ( $1 \mu \mathrm{g} / \mathrm{ml}, 6 \mathrm{hrs})$, cells became amoeboid like, numbers of filopodia-like processes increased, and lamellipodialike structures were enlarged and thickened (Fig. 2C). Interestingly, similar morphological changes were observed in OPNpt20 ( 0.5 or $1 \mu \mathrm{g} / \mathrm{ml})$-treated cells but not in OPNpt20-Db $(1 \mu \mathrm{g} / \mathrm{ml})$-treated cells (Fig. 2D and E). In addition, mean F-actin intensities and Factin-positive areas were significantly increased by OPNpt20 (1 $\mu \mathrm{g} / \mathrm{ml}$ ), to $184.3 \pm 12.0 \%$ and $175.2 \pm 19.1 \%$, respectively, versus PBStreated controls, which were $70.7 \pm 8.3 \%$ and $74.2 \pm 7.7 \%$ of those of rhOPN ( $1 \mu \mathrm{g} / \mathrm{ml}$ )-treated cells (Fig. $2 \mathrm{H}$ and I). Significant increases in mean F-actin intensities and F-actin-positive areas were also observed in FGF $(1 \mu \mathrm{g} / \mathrm{ml})$-treated cells but not in OPNpt20-Db $(1 \mu \mathrm{g} / \mathrm{ml})$ - or CytB $(1 \mu \mathrm{g} / \mathrm{ml})$-treated cells (Fig. 2B, F I). Together these results indicate OPNpt20 alters BV2 cell morphology and induces F-actin polymerization, and that the RGD and SLAYGLR motifs play important roles in these processes.

\section{OPNpt20-integrin interaction is involved in OPNpt20- mediated motility induction of BV2 cells}

To identify the signaling pathways underlying OPNpt20-mediated motility and morphological changes in BV2 cells, we first examined the interaction between OPNpt20 and endogenous av integrin. When pull-down assays were conducted after treating BV2 cells with biotinylated-OPNpt20, it was found exogenous OPNpt20 interacted with endogenous av integrin (Fig. 3A). Similarly, interaction between exogenous OPNpt20 and endogenous a4 integrin was also observed (Fig. 3B). To confirm the importance of integrin, BV2 cells were incubated with OPNpt20 in the presence of anti- $\alpha v$ antibody or soluble $\alpha v \beta 3$ integrin. Induction of cell motility by OPNpt20 was significantly suppressed in anti- 


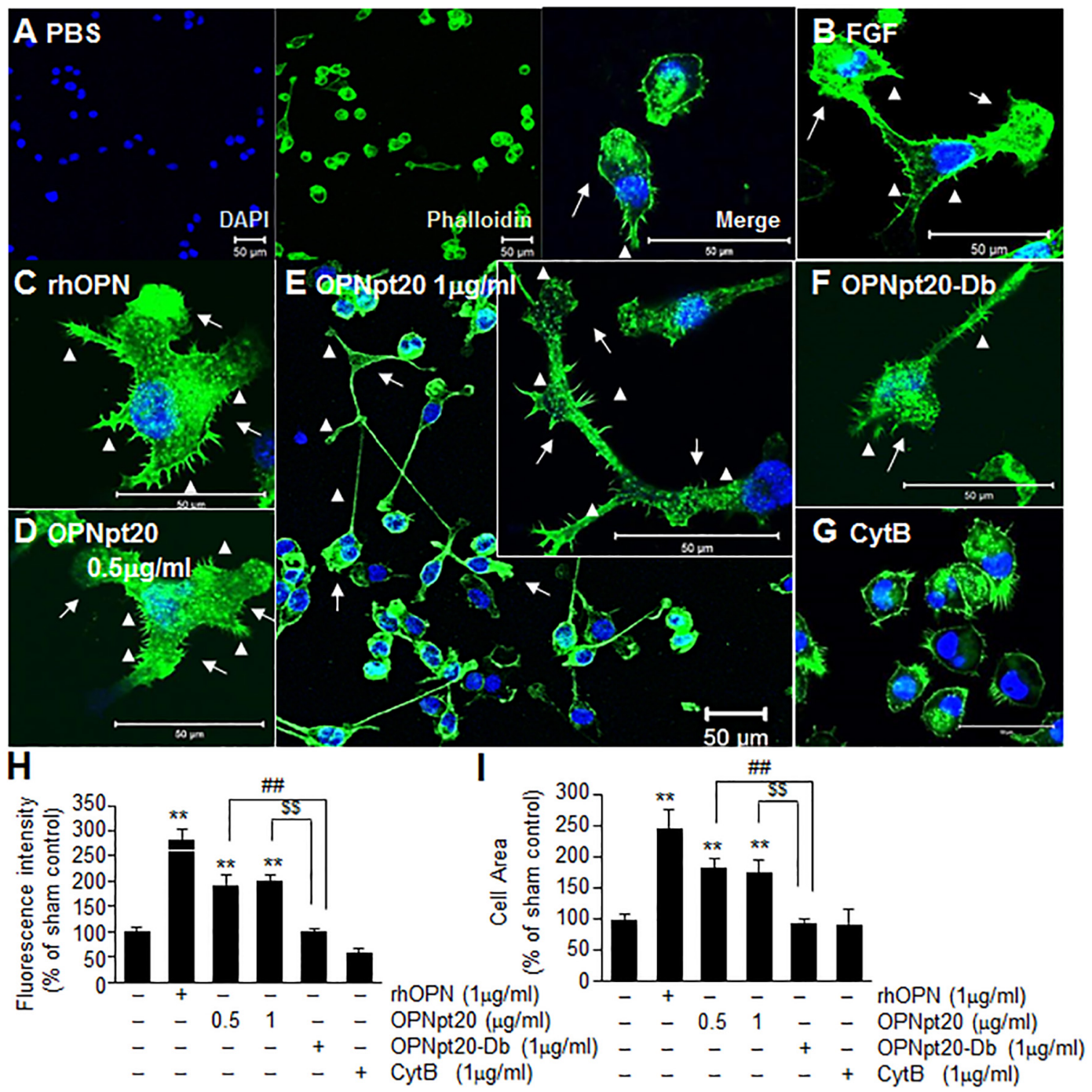

Fig. 2. Induction of F-actin polymerization by OPNpt20. BV2 cells were incubated with FGF (B, $1 \mu \mathrm{g} / \mathrm{ml})$, rhOPN (C; $1 \mu \mathrm{g} / \mathrm{ml}), \mathrm{OPNpt} 20$ (D, E; 0.5 or $1 \mu \mathrm{g} / \mathrm{ml})$, OPNpt20-Db (F; $1 \mu \mathrm{g} / \mathrm{ml}$ ), or CytB (G; $1 \mu \mathrm{g} / \mathrm{ml})$ for 6 hrs and then immunostained with FITC-labeled phalloidin. Fluorescence intensities (H) and microglial cell areas (I) were measured using Image J software, and results are presented as means \pm SEMs. Arrows indicate filopodia-like processes and arrowheads indicate lamellipodia-like structures. ${ }^{* *} \mathrm{p}<0.01$ versus PBS-treated controls and ${ }^{\# *} \mathrm{p}<0.01,{ }^{\$ S} \mathrm{p}<0.01$ between indicated groups. Scale bars represent $50 \mu \mathrm{m}$.

av antibody and soluble avß3 integrin co-treated cells (Fig. 3C G and Movie S4), indicating a crucial role of $\alpha v \beta 3$ integrin.

\section{OPNpt20 activated FAK-Erk and Akt signaling pathways}

Next, we examined the downstream signal pathways involved in OPNpt20-integrin interaction. After 1 hr of OPNpt20 treatment, phosphorylated-FAK, -Erk, and -Akt levels were significantly higher but these were not observed after treatment with OPNpt20$\mathrm{Db}$, suggesting the activations of these signaling pathways might be involved in OPNpt20-mediated cell changes (Fig. 4A C). Furthermore, increased F-actin polymerization by OPNpt20 was significantly suppressed by co-treating cells with PD98059 or wortmannin (pharmacological inhibitors of Erk and Akt, respectively) (Fig. 4D H). In addition, increased total migration distance by OPNpt20 was also significantly suppressed by co-treating PD98059 or wortmannin (Fig. 4I M and Movie S5). Together these results indicate that OPNpt20 enhanced BV2 cell motility by interacting with integrin(s) and subsequent activations of FAKErk and Akt signaling pathways.

\section{OPNpt20 enhanced zymosan-induced phagocytosis}

Since cell motility is an essential requirement for phagocytosis, we examined whether OPNpt20 enhanced the phagocytic activity of BV2 cells. When cells were stimulated with FITC- 

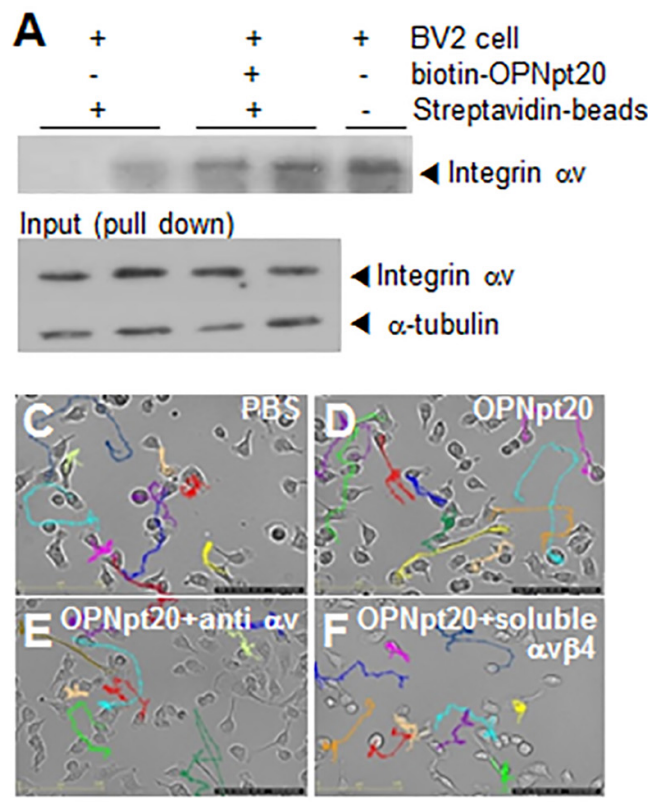

B

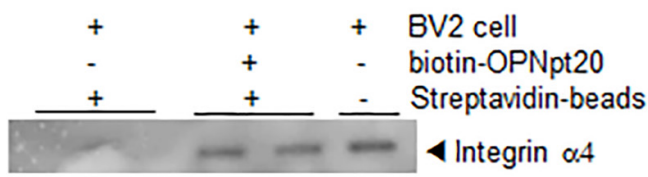

Input (pull down)
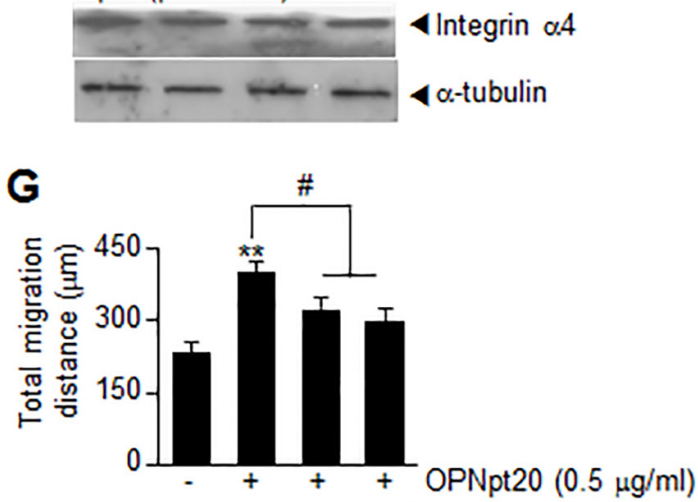

6 hrs after treatment

Fig. 3. OPNpt20 binding to av integrin in BV2 cells. (A, B) Biotinylated-OPNpt20 (1 $\mu \mathrm{g} / \mathrm{ml})$ was incubated with whole cell lysate of BV2 for 6 hrs and pull-down assays were performed using streptavidin agarose beads. Levels of av integrin (A) or a4 integrin (B) were assessed by immunoblotting. (C G) BV2 cells were incubated with OPNpt 20 for $6 \mathrm{hrs}$ in the presence of anti- $\alpha$ v integrin antibody $(0.5 \mu \mathrm{g} / \mathrm{ml})$ or soluble $\alpha v \beta 3 \mathrm{integrin}(0.5 \mu \mathrm{g} / \mathrm{ml})$. Cell motilities were measured every 10 minutes for 6 hrs by live cell imaging $(\mathrm{C} \sim \mathrm{F})$ and total migration distances were measured using Image J software $(\mathrm{G})$. Results are presented as means \pm SEMs. ${ }^{* *} \mathrm{p}<0.01$ versus PBS-treated controls and ${ }^{*} \mathrm{p}<0.05$ versus OPNpt20-treated cells. Scale bars represent $125 \mu \mathrm{m}(\mathrm{C} \sim \mathrm{F})$.
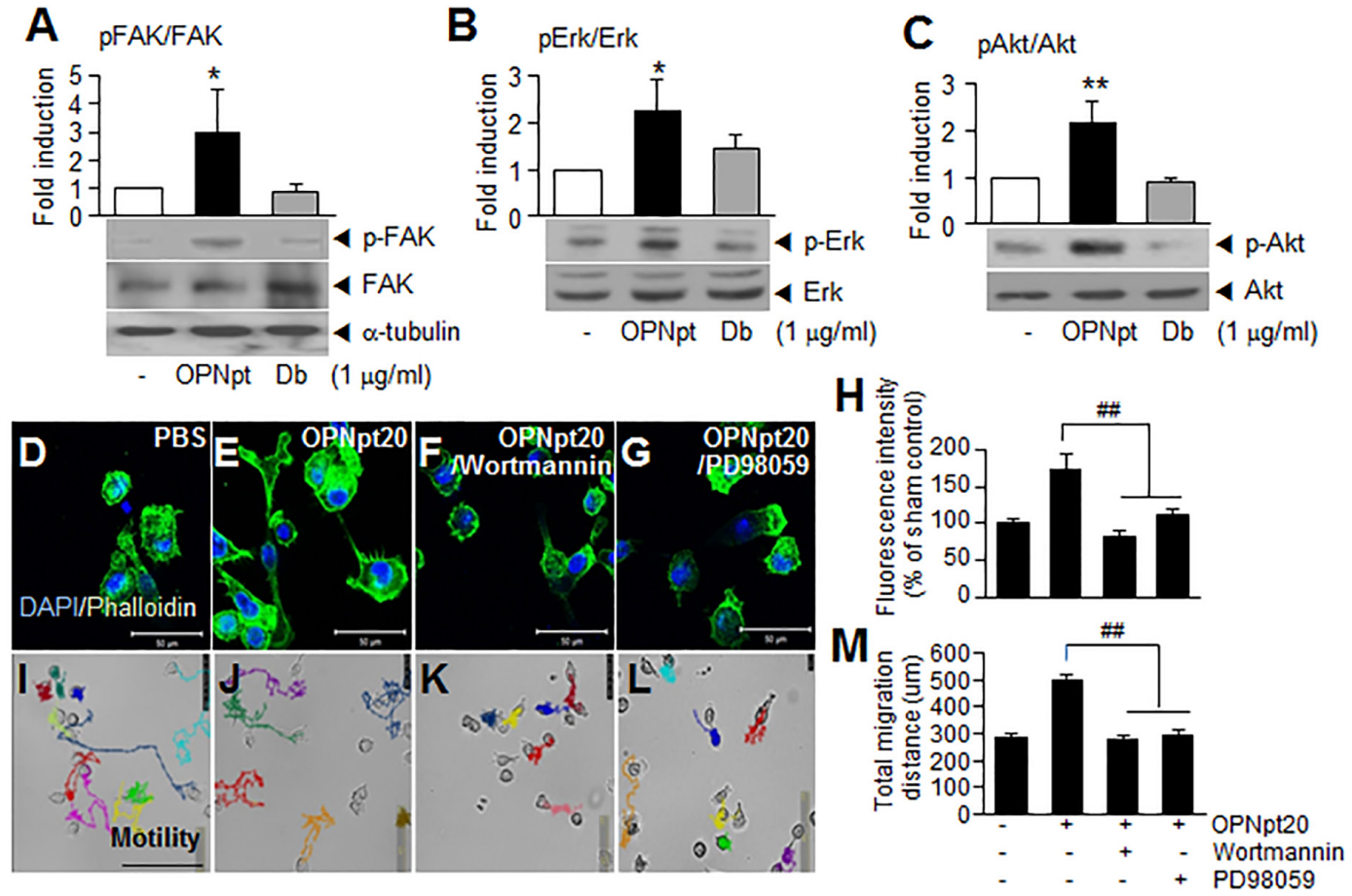

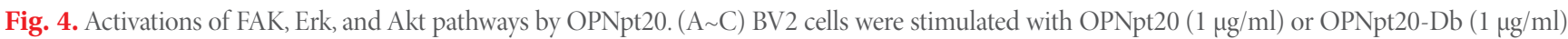
for $1 \mathrm{hr}$, and levels of phosphorylated or total FAK, Erk, and Akt were assessed by immunoblotting. (D M) BV2 cells were incubated with OPNpt20 for 6 hrs in the presence or absence of wortmannin $(0.2 \mu \mathrm{M}$; an Akt inhibitor) or PD 98059 (20 $\mu \mathrm{M}$; an Erk inhibitor). Cell morphologies were determined by image analysis using a confocal microscope $(\mathrm{D} \sim \mathrm{G})$ and cell motilities were measured every 10 minutes for 6 hrs by live cell imaging (I L). Fluorescence intensities $(\mathrm{H})$ and total migration distances $(\mathrm{M})$ were measured using Image J software and results are presented as means \pm SEMs. ${ }^{*} \mathrm{p}<0.05$, ${ }^{* *} \mathrm{p}<0.01$ versus PBS-treated controls and ${ }^{\# \#} \mathrm{p}<0.01$ versus OPNpt20-treated cells. Scale bars represent $50 \mu \mathrm{m}(\mathrm{D} \sim \mathrm{G})$ or $125 \mu \mathrm{m}$ (I L). 
labeled zymosan particles, which are microbial particles used to study phagocytic function, after 5 min of stimulation, zymosan particles were rapidly internalized. Numbers of internalized particles continuously increased throughout the 6 hrs observation period (Fig. 5A G), and numbers of zymosan-positive cells and of zymosan particles per cell also significantly increased (Fig. $5 \mathrm{H} \sim \mathrm{I})$. When BV2 cells were incubated with rhOPN $(1 \mu \mathrm{g} / \mathrm{ml})$ or OPNpt20 $(1 \mu \mathrm{g} / \mathrm{ml})$ in the presence of zymosan particles for $1 \mathrm{hr}$, internalized zymosan particles numbers increased to $146.9 \pm 8.9 \%$ or $132.4 \pm 11.5 \%$, respectively, of that of PBS controls (Fig. $6 \mathrm{~A} \sim \mathrm{E}$ and Movie S6), and similarly, numbers of zymosan particles per cell also increased to $131.5 \pm 10.6 \%$ or $113.3 \pm 3.4 \%$, respectively (Fig. 6A C, F, G and Movie S6). However, these increases were not observed in OPNpt20-Db-or CytB-treated cells (Fig. 6D G and Movie S6). Together these results show OPNpt20 enhanced phagocytosis by BV2 cells.

\section{DISCUSSION}

In previous studies, we reported OPNpt20 has robust neuroprotective and anti-inflammatory effects in the postischemic brain [13] and promote angiogenesis in HUVECs and in the postischemic rat brain [14]. In the present study, we found OPNpt20 induces the motility and phagocytic function of microglia. Although the induction of cell migration by OPN has been reported in various pathophysiological conditions, such as, in glioblastoma, breast cancer, acute lung injury, and stroke [2, 18, 23, 24], this is the first study to report that a 20-amino acid peptide of OPN containing RGD and SLAY motifs induces microglial phagocytotic activity in a RGD and SLAY motif-dependent manner.

Microglia are resident immune cell in the CNS, and under normal condition, they exert surveillance function for monitoring brain parenchyma and maintaining tissue homeostasis [16]. Under pathologic conditions, for example, in infectious diseases, microglial cells are activated and migrate to affected tissues to

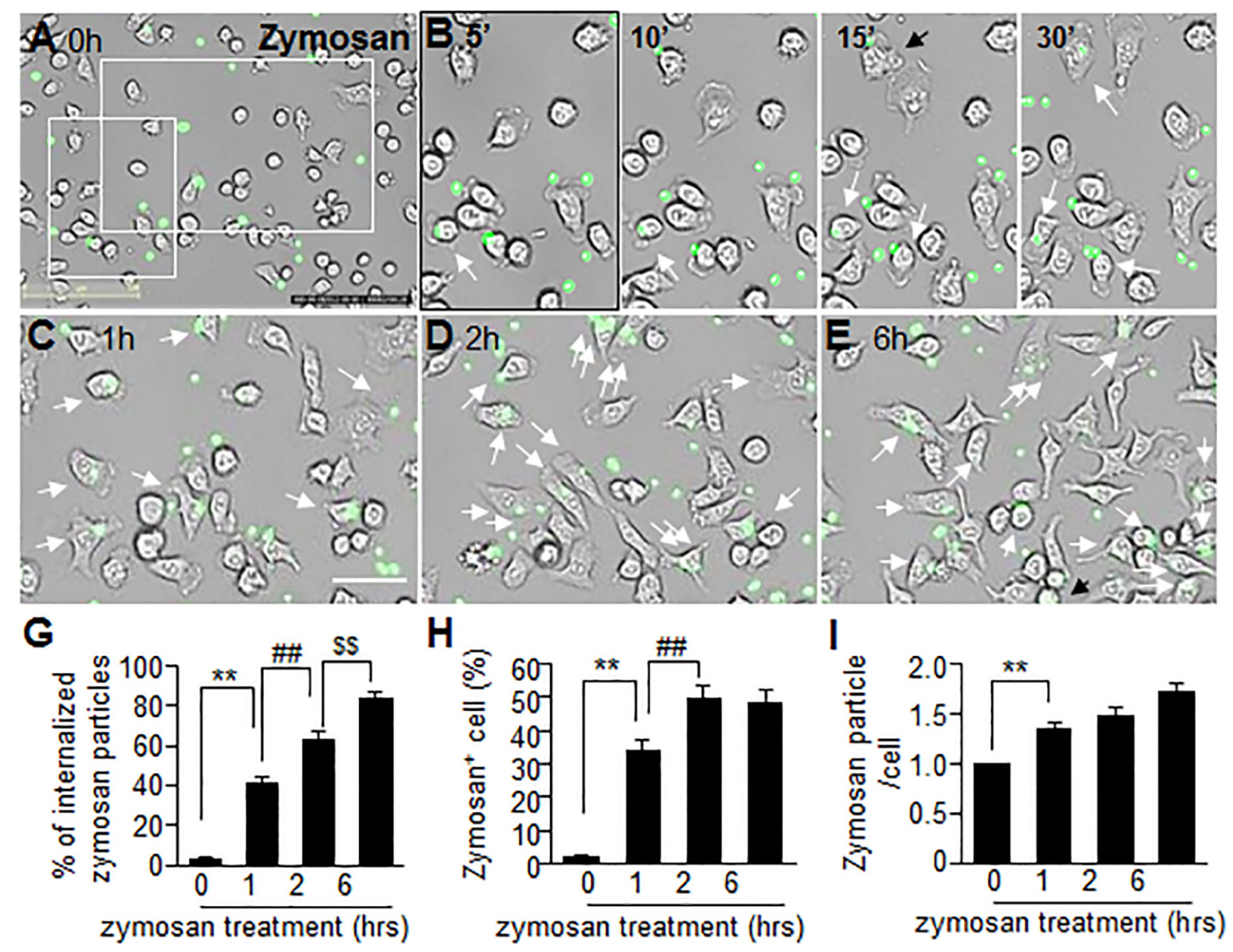

Fig. 5. Phagocytosis of zymosan particles by BV2 cells. BV2 cells were incubated with FITC-labeled zymosan particles and monitored by live cell imaging system for 12 hrs. Phagocytosis of zymosan particles was monitored every 5 minutes for 12 hrs (A H), and percentages of internalized zymosan particles (I), zymosan positive cells (J), and numbers of zymosan particles per cell (K) were determined by counting 10 photographic fields at $0,1,2,6$, and 12 hrs and results are presented as means \pm SEMs. Arrows indicate internalized zymosan particles. ${ }^{* *} \mathrm{p}<0.05$ versus PBS-treated control and ${ }^{* *} \mathrm{p}<0.01$, ${ }^{\$ \$} \mathrm{p}<0.01$ between indicated groups. Scale bars represent $125 \mu \mathrm{m}(\mathrm{A})$ or $50 \mu \mathrm{m}(\mathrm{B} \sim \mathrm{E})$. 


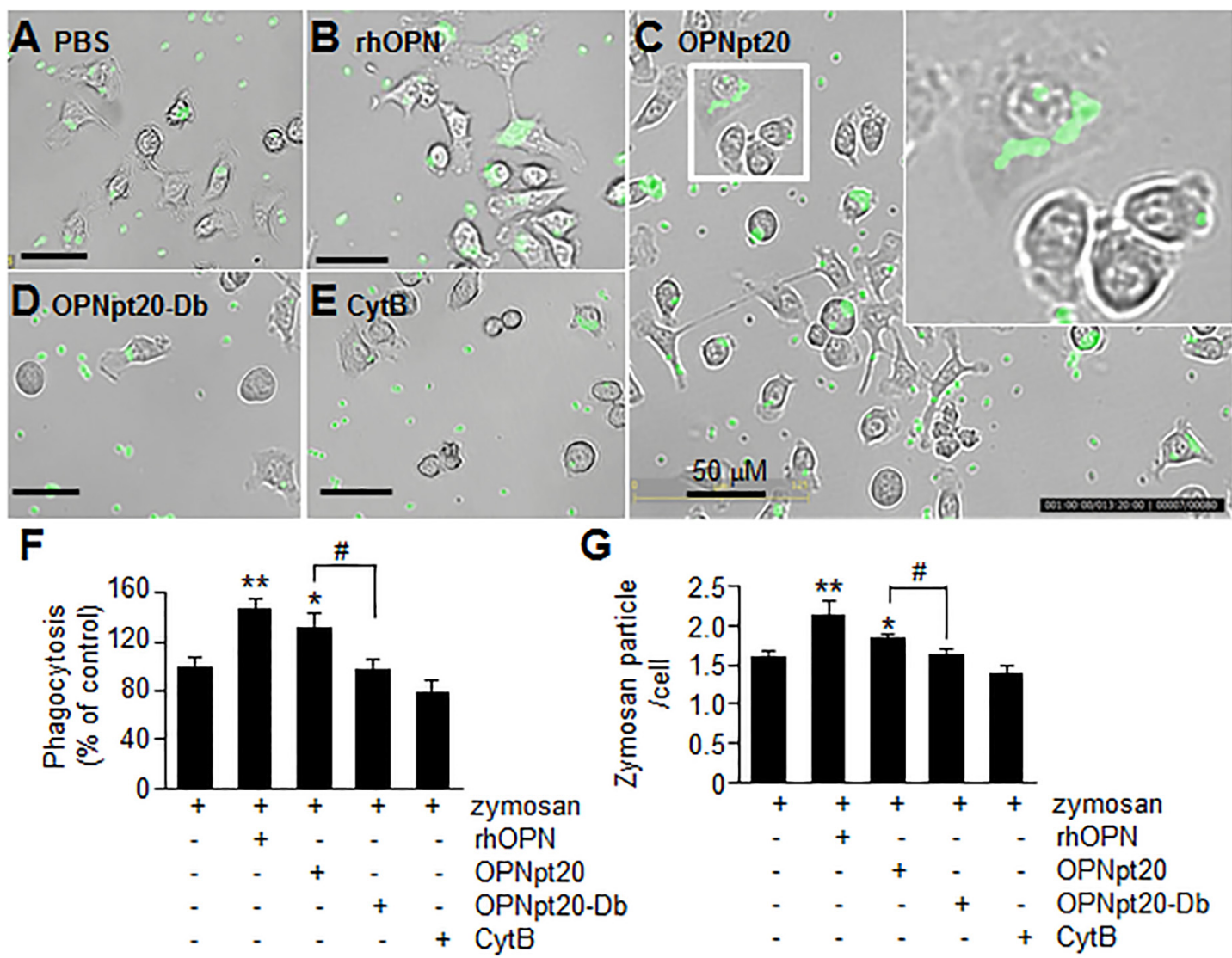

Fig. 6. Enhancement of zymosan phagocytosis by OPNpt20 in BV2 cells. BV2 cells were incubated with rhOPN (B; $1 \mu \mathrm{g} / \mathrm{ml})$, OPNpt20 (C; $1 \mu \mathrm{g} / \mathrm{ml})$, OPNpt20-Db (D; $1 \mu \mathrm{g} / \mathrm{ml})$, or CytB $(\mathrm{E} ; 1 \mu \mathrm{g} / \mathrm{ml})$ for $1 \mathrm{hr}$ in the presence of FITC-labeled zymosan particles and phagocytosis of zymosan particles was monitored every 5 minutes by live cell imaging analyzer. Percentages of internalized zymosan particles (F) and numbers of zymosan particles per cell (G) at $6 \mathrm{hrs}$ after zymosan stimulation were quantified by counting 10 photographic fields. Results are presented as means \pm SEMs. ${ }^{*} \mathrm{p}<0.05$, ${ }^{* *} \mathrm{p}<0.01 \mathrm{versus}$ zymosan-treated controls. ${ }^{*} \mathrm{p}<0.05$ versus OPNpt20-treated control. Scale bars represent $50 \mu \mathrm{m}$.

phagocytose infectious agents or cellular debris [16]. Similarly, in neurological diseases, such as, multiple sclerosis, Alzheimer's disease, and brain ischemia, activated microglia migrate to sites of injury and clear damaged neurons $[25,26]$. Accordingly, under pathological conditions, microglia must be motile to reach damaged areas [16], and this activation has been shown to be triggered by various chemoattractants, such as, small molecules, nucleotides, and bioactive lipids $[27,28]$. In the present study, OPNpt20 was found to enhance the motility and phagocytic activity of microglia, although slightly less than rOPN $(87.6 \pm 3.0 \%$ and $90.1 \pm 7.8 \%$ of rhOPN, respectively), which is known to induce the phagocytic activities and migrations of many cell types, including endothelial cells, neuroblasts, neutrophils and macrophages [2, 18, 19, 29]. The importance of the phagocytic activity of microglia has been reported in the postischemic brain, in which the phagocytosis of dead neurons is crucial for recovery and promotes axon regeneration and restoration of the microenvironment [30]. Therefore, it can be speculated that induction of phagocytic activity of mi- croglia might contribute to the robust neuroprotective effect of OPNpt20 shown in our previous report [13].

The RGD motif has been well documented to participate in various cellular functions, such as, migration, angiogenesis, and neuroprotection $[12,13,31]$, and the SLAY motif has also been reported to have protective effects in animal models of heart failure [32] and myocardial fibrosis [33]. In the present study, OPNpt20-RAA- or OPNpt20-SLAA-treated cells had comparable cell motilities, which were slightly, though significantly, lower than those of OPNpt20treated cells (Fig. 2). These results indicate that in the absence of a SLAY motif, RGD in OPNpt20 acts to induce cell motility in microglia, and that in the absence of the RGD motif, SLAY acts in a similar manner, but that when both motifs are present they act in an additive manner. Interestingly, the effects of the RGD and SLAY motifs of OPNpt20 are cell types and context-dependent, for example, SLAY appeared to play more important roles in the inductions of cell motility and phagocytic activity of microglia than in the induction of anti-inflammatory effects [13]. Although, we 
observed endogenous $\alpha_{v}$ integrin bound to OPNpt20 to exert antiinflammatory and pro-angiogenic effects $[13,14]$ and endogenous $\alpha_{v}$ and $\alpha_{4}$ and integrins bound to OPNpt20 to exert inductions of cell motility (Fig. 3A and B), it is quite possible other integrin also bind to OPNpt20, and that different downstream signaling pathways might be responsible for the different effects of this peptide.

The potential therapeutic applications of OPN have been investigated in various pathological contexts, and exogenously administered recombinant OPN protein has been reported to have protective effects in a mouse model of transient focal ischemia [5], in intracerebral hemorrhage mouse model [3], and in animal models of Parkinson's disease [4], and a neonatal hypoxia-ischemia induced brain injury [6]. Recently, we reported recombinant OPN (rOPN) protein had robust neuroprotective effects in the postischemic brain, and that this effect was enhanced by delivering rOPN in biodegradable gelatin microspheres [7]. Gilbert [34] reported that a 21 -amino-acid OPN peptide harboring hydroxyapatite binding domain and a RGD motif mediated $\alpha_{\mathrm{v}}$ integrin adhesion in melanoma cells. Furthermore, Doyle [11] found that a 20or even a 10-amino-acid OPN peptide containing the RGD and SLAYGLR motifs had neuroprotective effect in an animal model of transient focal ischemia and Mizuno [35] reported a 7-aminoacid OPN peptide harboring the SVVYGLR sequence improved cardiac function in a hamster model of dilated cardiomyopathy. These reports consistently suggest short OPN-derived peptides containing the RGD motif and the SLAYGLR sequence have neuroprotective effects.

In the present study, we reported OPNpt20 is a multifunctional peptide by adding its phagocytosis-inducing activity to two functions previously shown, which are anti-inflammatory [13] and pro-angiogenic effects [14]. In view of the multifunctionality of OPN, it would be interesting to determine whether OPNpt20 has additional functions, especially functions associated for OPN, such as the scavenging of excess $\mathrm{Ca}^{2+}$. It appears OPNpt20 has considerable potential in the context of treating diseases related to above-mentioned functions.

\section{ACKNOWLEDGEMENTS}

This work was supported by a Mid-carrier Research Grant (2015R1A2A2A01003395) funded by the National Research Foundation (NRF) of Korea (to J.-K.L.) and a Basic Science Research Program through the National Research Foundation of Korea (NRF) funded by the Ministry of Education (2015R1D1A4A01020716) (to I.-D.K.).

\section{REFERENCES}

1. Rittling SR (2011) Osteopontin in macrophage function. Expert Rev Mol Med 13:e15.

2. Yan YP, Lang BT, Vemuganti R, Dempsey RJ (2009) Osteopontin is a mediator of the lateral migration of neuroblasts from the subventricular zone after focal cerebral ischemia. Neurochem Int 55:826-832.

3. Suzuki H, Hasegawa Y, Kanamaru K, Zhang JH (2010) Mechanisms of osteopontin-induced stabilization of blood-brain barrier disruption after subarachnoid hemorrhage in rats. Stroke 41:1783-1790.

4. Ailane S, Long P, Jenner P, Rose S (2013) Expression of integrin and CD44 receptors recognising osteopontin in the normal and LPS-lesioned rat substantia nigra. Eur J Neurosci 38:2468-2476.

5. Schroeter M, Zickler P, Denhardt DT, Hartung HP, Jander S (2006) Increased thalamic neurodegeneration following ischaemic cortical stroke in osteopontin-deficient mice. Brain 129:1426-1437.

6. Chen W, Ma Q, Suzuki H, Hartman R, Tang J, Zhang JH (2011) Osteopontin reduced hypoxia-ischemia neonatal brain injury by suppression of apoptosis in a rat pup model. Stroke 42:764-769.

7. Jin Y, Kim IY, Kim ID, Lee HK, Park JY, Han PL, Kim KK, Choi H, Lee JK (2014) Biodegradable gelatin microspheres enhance the neuroprotective potency of osteopontin via quick and sustained release in the post-ischemic brain. Acta Biomater 10:3126-3135.

8. Standal T, Borset M, Sundan A (2004) Role of osteopontin in adhesion, migration, cell survival and bone remodeling. Exp Oncol 26:179-184.

9. Yokosaki Y, Matsuura N, Sasaki T, Murakami I, Schneider H, Higashiyama S, Saitoh Y, Yamakido M, Taooka Y, Sheppard D (1999) The integrin alpha(9)beta(1) binds to a novel recognition sequence (SVVYGLR) in the thrombin-cleaved aminoterminal fragment of osteopontin. J Biol Chem 274:3632836334.

10. Bayless KJ, Meininger GA, Scholtz JM, Davis GE (1998) Osteopontin is a ligand for the alpha4betal integrin. J Cell Sci 111:1165-1174.

11. Doyle KP, Yang T, Lessov NS, Ciesielski TM, Stevens SL, Simon RP, King JS, Stenzel-Poore MP (2008) Nasal administration of osteopontin peptide mimetics confers neuroprotection in stroke. J Cereb Blood Flow Metab 28:1235-1248.

12. Iczkiewicz J, Broom L, Cooper JD, Wong AM, Rose S, Jenner P (2010) The RGD-containing peptide fragment of osteo- 
pontin protects tyrosine hydroxylase positive cells against toxic insult in primary ventral mesencephalic cultures and in the rat substantia nigra. J Neurochem 114:1792-1804.

13. Jin YC, Lee H, Kim SW, Kim ID, Lee HK, Lee Y, Han PL, Lee JK (2016) Intranasal delivery of RGD motif-containing osteopontin icosamer confers neuroprotection in the postischemic brain via av $\beta 3$ Integrin integrin binding. Mol Neurobiol 53:5652-5663.

14. Lee H, Jin YC, Kim SW, Kim ID, Lee HK, Lee JK (2017) Proangiogenic functions of RGD-SLAY-containing osteopontin icosamer peptide in HUVECs and the postischemic brain. Exp Mol Med (in press).

15. Aguzzi A, Barres BA, Bennett ML (2013) Microglia: scapegoat, saboteur, or something else? Science 339:156-161.

16. Garden GA, Möller T (2006) Microglia biology in health and disease. J Neuroimmune Pharmacol 1:127-137.

17. Bollmann L, Koser DE, Shahapure R, Gautier HO, Holzapfel GA, Scarcelli G, Gather MC, Ulbricht E, Franze K (2015) Microglia mechanics: immune activation alters traction forces and durotaxis. Front Cell Neurosci 9:363.

18. Hirano Y, Aziz M, Yang WL, Wang Z, Zhou M, Ochani M, Khader A, Wang P (2015) Neutralization of osteopontin attenuates neutrophil migration in sepsis-induced acute lung injury. Crit Care 19:53.

19. Weber GF, Zawaideh S, Hikita S, Kumar VA, Cantor H, Ashkar S (2002) Phosphorylation-dependent interaction of osteopontin with its receptors regulates macrophage migration and activation. J Leukoc Biol 72:752-761.

20. Schack L, Stapulionis R, Christensen B, Kofod-Olsen E, Skov Sørensen UB, Vorup-Jensen T, Sørensen ES, Höllsberg P (2009) Osteopontin enhances phagocytosis through a novel osteopontin receptor, the alphaXbeta2 integrin. J Immunol 182:6943-6950.

21. Heilmann K, Hoffmann U, Witte E, Loddenkemper C, Sina C, Schreiber S, Hayford C, Holzlöhner P, Wolk K, Tchatchou E, Moos V, Zeitz M, Sabat R, Günthert U, Wittig BM (2009) Osteopontin as two-sided mediator of intestinal inflammation. J Cell Mol Med 13:1162-1174.

22. Cross MJ, Claesson-Welsh L (2001) FGF and VEGF function in angiogenesis: signalling pathways, biological responses and therapeutic inhibition. Trends Pharmacol Sci 22:201-207.

23. Ellert-Miklaszewska A, Wisniewski P, Kijewska M, Gajdanowicz P, Pszczolkowska D, Przanowski P, Dabrowski M, Maleszewska M, Kaminska B (2016) Tumour-processed osteopontin and lactadherin drive the protumorigenic reprogramming of microglia and glioma progression. Oncogene 35:6366-6377.
24. Ortiz-Martínez F, Sanmartín E, Pomares-Navarro E, PérezBalaguer A, Andrés L, Sánchez-Payá J, Aranda FI, Lerma E, Peiró G (2015) Osteopontin regulates VEGFA and ICAM1 mRNA expression in breast carcinoma. Am J Clin Pathol 143:812-822.

25. Fu R, Shen Q, Xu P, Luo JJ, Tang Y (2014) Phagocytosis of microglia in the central nervous system diseases. Mol Neurobiol 49:1422-1434.

26. Prinz M, Priller J (2014) Microglia and brain macrophages in the molecular age: from origin to neuropsychiatric disease. Nat Rev Neurosci 15:300-312.

27. Bianchi R, Kastrisianaki E, Giambanco I, Donato R (2011) S100B protein stimulates microglia migration via RAGE-dependent up-regulation of chemokine expression and release. J Biol Chem 286:7214-7226.

28. Wang S, Chu CH, Stewart T, Ginghina C, Wang Y, Nie H, Guo M, Wilson B, Hong JS, Zhang J (2015) a-Synuclein, a chemoattractant, directs microglial migration via $\mathrm{H} 2 \mathrm{O} 2$ dependent Lyn phosphorylation. Proc Natl Acad Sci U S A 112:E1926-E1935.

29. Senger DR, Ledbetter SR, Claffey KP, Papadopoulos-Sergiou A, Peruzzi CA, Detmar M (1996) Stimulation of endothelial cell migration by vascular permeability factor/vascular endothelial growth factor through cooperative mechanisms involving the alphavbeta3 integrin, osteopontin, and thrombin. Am J Pathol 149:293-305.

30. Ito U, Nagasao J, Kawakami E, Oyanagi K (2007) Fate of disseminated dead neurons in the cortical ischemic penumbra: ultrastructure indicating a novel scavenger mechanism of microglia and astrocytes. Stroke 38:2577-2583.

31. Takahashi K, Takahashi F, Tanabe KK, Takahashi H, Fukuchi Y (1998) The carboxyl-terminal fragment of osteopontin suppresses arginine-glycine-asparatic acid-dependent cell adhesion. Biochem Mol Biol Int 46:1081-1092.

32. Uchinaka A, Kawaguchi N, Hamada Y, Mori S, Miyagawa S, Saito A, Sawa Y, Matsuura N (2013) Transplantation of myoblast sheets that secrete the novel peptide SVVYGLR improves cardiac function in failing hearts. Cardiovasc Res 99:102-110.

33. Uchinaka A, Hamada Y, Mori S, Miyagawa S, Saito A, Sawa Y, Matsuura N, Yamamoto H, Kawaguchi N (2015) SVVYGLR motif of the thrombin-cleaved N-terminal osteopontin fragment enhances the synthesis of collagen type III in myocardial fibrosis. Mol Cell Biochem 408:191-203.

34. Gilbert M, Shaw WJ, Long JR, Nelson K, Drobny GP, Giachelli CM, Stayton PS (2000) Chimeric peptides of statherin and osteopontin that bind hydroxyapatite and mediate cell adhe- 
sion. J Biol Chem 275:16213-16218.

35. Mizuno Y, Uchinaka A, Horii Y, Mori S, Hamada Y, Miyagawa S, Saito A, Sawa Y, Matsuura N, Kawaguchi N (2015) Improvement of cardiac function after implanting the osteopontin-derived peptide SVVYGLR in a hamster model of dilated cardiomyopathy. Interact Cardiovasc Thorac Surg 21:506-514. 


\section{Supplementary Figure 1.}
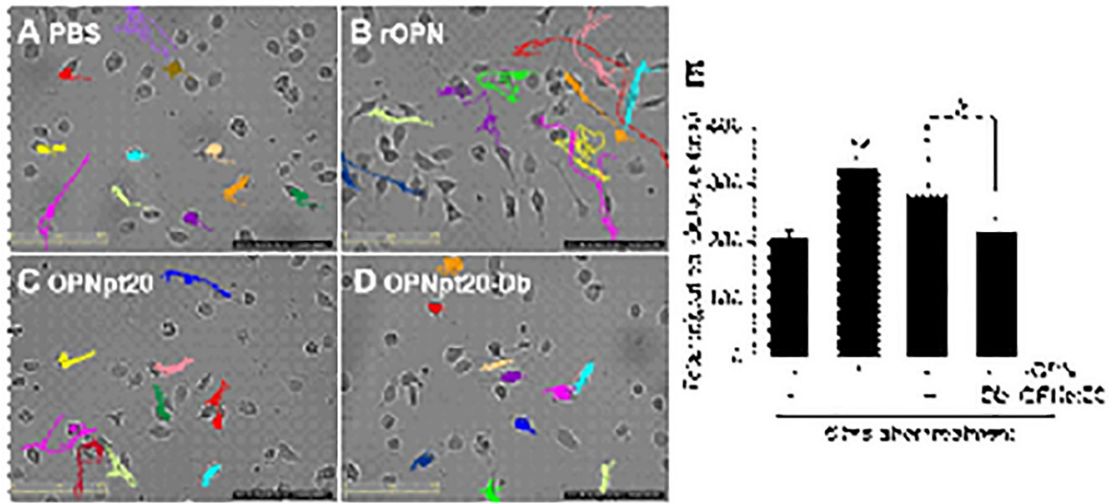

Supplem entary Figure 1. Induction of cell motility by OPNpt20 in primary

microglial culture

Primary microglia cells were incubated with $\operatorname{rhOPN}(\mathrm{B}, 1 \mu \mathrm{g} / \mathrm{ml})$, OPNpt20 (C, 1 $\mu \mathrm{g} / \mathrm{ml})$, or OPNpt $20-\mathrm{Db}(\mathrm{D}, 1 \mu \mathrm{g} / \mathrm{ml})$ for $6 \mathrm{hrs}$ and cell motilities were monitored using a live cell imaging analyzer at every $6 \mathrm{~min}$. Motile trajectory of each cell after $6 \mathrm{hrs}$ are presented (A-D) and mean total migration distances over $6 \mathrm{hrs}(\mathrm{E})$ are presented as means \pm SEMs $(n=10) .{ }^{*} p<0.05, *{ }^{*} p<0.01$ versus PBS-treated controls, $\# p<0.05$ versus OPNpt20-treated controls. Scale bars represent $125 \mu \mathrm{m}$. 